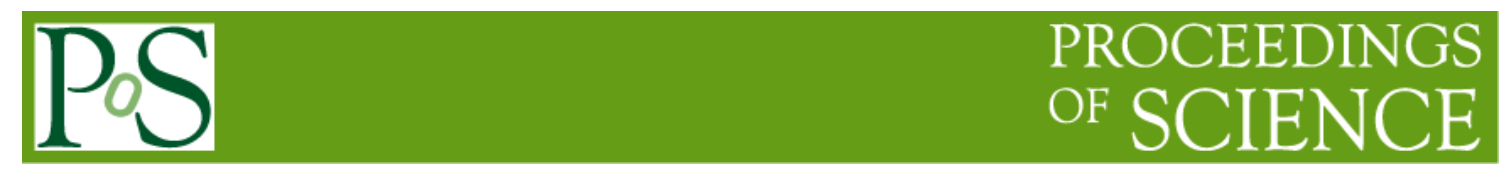

\title{
Polarized ion source with nearly resonant charge- exchange plasma ionizer: parameters and possibilities for improvements
}

\author{
A. S. Belov \\ Institute for Nuclear Research of Russian Academy of Sciences \\ Moscow, 117312, Russia \\ E-mail: beloveinr.ru
}

\begin{abstract}
Parameters of polarized ion sources with nearly resonant charge-exchange plasma ionizer are summarized. Pulsed beams of polarized protons with peak intensity up to $11 \mathrm{~mA}$ and polarization of $80 \%$ and polarized negative hydrogen ions with peak current of $4 \mathrm{~mA}$ and polarization of $91 \%$ have been obtained. While no progress of parameters during recent past years has been obtained, possibilities for further improvements still exist. Both atomic beam parts of the sources can be improved as well as efficiency of conversion of polarized atoms into polarized ions. The possibilities are discussed in the paper.
\end{abstract}

XVth International Workshop on Polarized Sources, Targets, and Polarimetry September 9-13, 2013

Charlottesville, Virginia, USA 


\section{Introduction}

Development of high intensity sources of pulsed polarized ion beams was inspired since 1980th by proposed spin physics experiments. Sources of polarized negative hydrogen ions with peak intensity of $\sim 1 \mathrm{~mA}$ and high polarization were necessary for projects of acceleration of polarized protons in high energy machines.

The goal has been achieved with development of high intensity atomic beam-type source (ABS) with resonant charge-exchange plasma ionizer [1] and optically pumped polarized ion sources (OPPIS) [2]. These type sources produce polarized negative hydrogen ions with current of few milliamperes, high polarization and acceptable emittance. Characteristics of the ABS with nearly resonant charge-exchange plasma ionizer are summarized in chapter 2 of the paper. Possibilities for further improvement of the source are presented in chapter 3.

\section{Characteristics of the ABS with nearly resonant charge-exchange plasma ionizer}

The ABS with nearly resonant charge-exchange plasma ionizer has been developed at INR RAS (see [2] and herein). In the source polarized protons or polarized $\mathrm{H}$ - ions are produced via charge exchange collisions between polarized hydrogen atoms and unpolarized deuterium ions in deuterium plasma.

Polarized hydrogen atoms are produced with an atomic beam apparatus consisting of an RF discharge hydrogen dissociator, sextupole electromagnets and an RF transition unit.

The pulsed polarized atomic hydrogen beam has peak intensity $\sim 2 \cdot 10^{17} \mathrm{at} / \mathrm{cm}^{2} \mathrm{sec}$ downstream the sextupole magnets at the plasma ionizer entrance.

Polarized protons are produced in the plasma ionizer with free polarized atomic hydrogen beam injected into plasma or with a storage cell installed into the charge-exchange region. Polarized proton beam with peak current up to $6 \mathrm{~mA}$, pulse duration (FWHM) of $\sim 100 \mu \mathrm{s}$, repetition rate up to $10 \mathrm{~Hz}$ and normalized emittance of $\sim 2 \pi \mathrm{mm}$ mrad has been obtained from the source with free polarized atomic hydrogen beam [3]. Unpolarized deuteron current density transported through the charge-exchange region was $\sim 250 \mathrm{~mA} / \mathrm{cm}^{2}$ at the extraction system of the ionizer.

Proton polarization has been increased from 0.76 to 0.9 due to installation of a cold trap around the charge-exchange region and reducing by this way of unpolarized background proton current extracted from plasma [4].

Installation of a storage cell into the plasma ionizer leads to increase of density of polarized hydrogen atoms in the charge-exchange region. When the storage cell made from aluminum alloy was installed into the charge-exchange region the polarized gaseous target thickness formed by polarized hydrogen atoms for unpolarized deuterium ions in plasma increased one order of magnitude in comparison with free atomic hydrogen beam [5]. However, it was found that transport of a plasma flux through the storage cell is restricted due to plasma instabilities. It was found that "noiseless" mode of a plasma source operation is important for the plasma transport through the storage cell as well as increase of longitudinal magnetic field in 
the charge - exchange region. With these measures unpolarized $\mathrm{D}^{+}$ion flux with pulsed current of $45 \mathrm{~mA}$ was transported through the storage cell.

Respectively, polarized proton current with peak intensity up to $11 \mathrm{~mA}$, polarization of 0.8 , pulse duration of $200 \mu \mathrm{s}$ at repetition rate of $10 \mathrm{~Hz}$ has been obtained [6]. The normalized emittance was $\sim 1 \pi \mathrm{mm}$ mrad. Brightness of the polarized beam was almost one order of magnitude larger than the brightness of polarized proton beam obtained from the source without the storage cell.

For production of polarized negative hydrogen ions in the same source deuterium plasma was enriched with unpolarized $\mathrm{D}^{-}$ions. For that an injector of deuterium plasma with surfaceplasma convertor of plasma particles into the $\mathrm{D}^{-}$ions has been developed [7]. The injector generates deuterium plasma consisting mainly from $\mathrm{D}^{+}$and $\mathrm{D}^{-}$ions with low density of electrons. This is important feature of the injector because otherwise destruction of polarized $\mathrm{H}^{-}$ ions in collisions with plasma electrons will limit the polarized $\mathrm{H}^{-}$ion current. Finally, after multistep development unpolarized $\mathrm{D}^{-}$ion beam with peak current of $60 \mathrm{~mA}$ was obtained from the plasma ionizer with plasma injector developed. Polarized $\mathrm{H}^{-}$ion beam with peak intensity of $4 \mathrm{~mA}$ and polarization of $\sim 0.9$ has been obtained from the source [1].

In spite of high intensity of polarized proton and $\mathrm{H}^{-}$ion beams achieved, there are possibilities for further improvements of the polarized ion source.

\section{Possibilities for improvements of the polarized ion source parameters}

\subsection{Atomic beam apparatus optimization}

Intensity of the polarized ion source is proportional to target thickness formed by polarized atomic hydrogen beam injected into the charge-exchange region for plasma ions.

The target thickness formed by polarized atoms is proportional to the atomic beam intensity which is limited in the INR RAS polarized ion source to value of $\sim 2 \cdot 10^{17} \mathrm{at} / \mathrm{cm}^{2} \mathrm{sec}$ due to effect of beam-skimmer interference and noncomplete cooling of hydrogen atoms in the pulsed RF discharge dissociator [1].

Measurements of radial size of hydrogen atoms source reported in [8] show increase of the radial size of the atoms source vs gas flux. Moreover, it was found that the radial distribution of hydrogen atoms density in the source has tails with size larger than the skimmer radius. It was supposed that the tails originate due to gaseous "cloud" formation at the skimmer orifice and inside the skimmer.

The atoms radial distribution in the source evidently makes strong influence on focusing properties of separating magnets system of ABS. For optimization of the separating magnets system it is desirable to have realistic parameters of the atoms source including not only radial distribution but also distribution of hydrogen atoms source in a transversal phase plane. The transversal emittance measurement is standard procedure for ion sources but it was not used yet for atomic hydrogen sources.

We plan to perform the transversal emittance measurements for atomic hydrogen source using well-known two slits method and movable mass-spectrometer for detection of hydrogen atoms. 
The transversal emittance measurements together with velocity distribution measurements should produce full necessary information concerning the hydrogen atoms source parameters and make possible optimization of the beam forming system parameters such as nozzleskimmer distance, skimmer orifice diameter etc.

Next step should be design of new separating magnet system. The INR ABS has two electromagnet sextupole with magnetic field at poles tips of $0.9 \mathrm{kG}$ while modern materials make possible production of permanent magnet sextupole with pole tip of $\sim 1.7 \mathrm{~T}$. Taking into account that acceptance angle of sextupole magnets is directly proportional to magnetic field at pole tip we could expect gain factor of $\sim 2$ in the atomic hydrogen beam intensity due to the optimization of the ABS apparatus.

Increase of polarized atomic hydrogen beam intensity will produce also some increase of ion beam polarization due to reducing dilution factor connected with unpolarized ion production in plasma ionizer (roughly from present factor of 0.9-0.95 to value of 0.95-0.97 respectively).

\subsection{Plasma ionizer optimization}

Intensity of polarized $\mathrm{H}+(\mathrm{H}-)$ beam from the source is proportional also to unpolarized $\mathrm{D}+\left(\mathrm{D}^{-}\right)$ion flux which is transported through the charge-exchange region of the polarized ion source.

For polarized proton source with free polarized atomic hydrogen beam in the ionizer the pulsed unpolarized $\mathrm{D}^{+}$flux through the charge-exchange region is limited mainly by properties of an ion extraction system. Achieved $\mathrm{D}^{+}$ion flux density of $250 \mathrm{~mA} / \mathrm{cm}^{2}$ [3] is close to a limit determined by voltage breakdowns in the extraction gap of the ion-optical system.

However, for polarized proton source with a storage cell in the ionizer plasma ion flux was limited to value of $45 \mathrm{~mA}$ by origination of "noise" mode of plasma flux. It was found that increase of magnetic field in the charge-exchange region allows increasing the transported ion flux through the storage cell [6]. So, increase of magnetic field in the plasma ionizer should allow increase of polarized ion beam current as well. Taking into account that emittance of polarized ion beam is proportional to magnetic field in the ionizer we can conclude that only moderate gain $\sim 2$ is possible due to this ionizer development. Another possibility is connected with cooling of the storage cell walls and minimization of atoms recombination.

Some increase of polarized $\mathrm{H}$ - ion beam current also can be obtained due to increase of unpolarized D- ion flux through the charge-exchange region. However, in this case the development should be made together with relative decrease of electron density in deuterium plasma to restrict destruction of polarized $\mathrm{H}$ - ions in collisions with plasma electrons.

\section{Conclusions}

ABS with nearly resonant charge-exchange plasma ionizer developed at INR RAS produces pulsed high intensity and high polarization beams of polarized protons and $\mathrm{H}$ - ions.

Polarized proton beam with peak current of $11 \mathrm{~mA}$ and polarization of 0.8 has been obtained with the storage cell installed in the charge-exchange region of the source plasma 
ionizer. For production of polarized $\mathrm{H}^{-}$ions injector of deuterium plasma consisting mainly from D+ and D- ions has been developed. Polarized H- ion beam with peak current of $4 \mathrm{~mA}$ and polarization of $\sim 0.9$ was obtained.

The source intensity can be increased due to atomic beam apparatus improvement. It is proposed to measure hydrogen atom source transversal emittance to have data for optimization of atomic beam formation and separating sextupole magnet system.

Another possibility for the source improvement is increase of unpolarized ion flux through the charge-exchange region of the plasma ionizer.

With these improvements it seems to be possible to achieve intensity of $\mathrm{H}$ - ion beam of $\sim 10 \mathrm{~mA}$ and polarized protons of $\sim 20 \mathrm{~mA}$ with high polarization of $\sim 0.9$.

\section{References}

[1] A. S. Belov, "Production of polarized ions in nearly resonant charge-exchange collisions in plasma”, in Proc. of 12th International Workshop on Polarized Sources Targets and Polarimeters, PSTP 2007, BNL, USA, 10-14 September, 2007, AIP Conf. Proc. 980, pp. 209-220, (2008).

[2] A. N. Zelenski, this Workshop.

[3] A. S. Belov, S. K. Esin, S. A. Kubalov, V. E. Kuzik, A. A. Stepanov, V. P. Yakushev, Nucl. Instrum. Meth., A255, 442-459 (1987).

[4] S. P. Agafonov, A. S. Belov, S. A. Kubalov, V. E. Kuzik, S. Yu. Merkulov, E. E. Nesterenko, Pribori i Tekhnika Eksperimenta, 4, 159-163 (1990).

[5] A. S. Belov, S. K. Esin, L. P. Netchaeva, V. S. Klenov, A. V. Turbabin, and G. A. Vasil'ev, "Polarized ions from a storage cell", in Proc. of Seventh International Workshop on Polarized Gas Targets and Polarized Beams", Urbana, IL, 1997, eds. Roy J. Holt and M.A. Miller, AIP Conf. Proc. 421, 362-371 (1998).

[6] A. S. Belov, S. K. Esin, L. P. Netchaeva, A. V. Turbabin, and G. A. Vasil'ev, "Polarized Ion Source with Resonant Charge-Exchange Plasma Ionizer", in Proc. of $13^{\text {th }}$ International Symposium on High Energy Spin Physics, Protvino, Russia, 9-12 September, 1998, eds. N.E. Tyurin et.al., World Scientific, 1999, p.p. 622-624.

[7] A. S. Belov, V. G. Dudnikov, V. E. Kuzik, Yu. V. Plokhinsky, V. P. Yakushev, Nucl. Instrum. Meth., A333, 256-259 (1993).

[8] A. S. Belov, R. Gebel, "Measurements of radial size of a hydrogen atoms source”, Proc. of $X I V^{\text {th }}$ International Workshop on Polarized Sources, Targets \& Polarimetry, St. Petersburg, 12-16 September, Russia, ISBN 978-5-86763-282-3, p.p. 69-72. 